\title{
OPTIMAL CONTROL OF LINEAR BOTTLENECK PROBLEMS
}

\author{
M. BERGOUNIOUX AND F. TRÖLTZSCH
}

\begin{abstract}
The regularity of Lagrange multipliers for state-constrained optimal control problems belongs to the basic questions of control theory. Here, we investigate bottleneck problems arising from optimal control problems for PDEs with certain mixed control-state inequality constraints. We show how to obtain Lagrange multipliers in $L^{p}$-spaces for linear problems and give an application to linear parabolic optimal control problems.
\end{abstract}

\section{INTRODUCTION}

The regularity of Lagrange multipliers for state-constrained optimal control problems belongs to the basic questions of control theory. This is known for control systems governed by ODEs and refers to PDEs as well. In many cases, Lagrange multipliers have to be measures, for instance, if pointwise state-constraints are given in distributed parameter systems. Unfortunately, several methods cannot be applied for measures as Lagrange multipliers. In particular, standard second order sufficient optimality conditions cannot be established, and the convergence of some numerical techniques is not ensured.

It is well known that Lagrange multipliers can be obtained for nonlinear programming problems in Banach spaces (including control problems) by considering the associated linearized problem (see Zowe and Kurcyusz [15]). In this way, the theory of linear programming problems in Banach spaces may serve as a tool to prove the existence of Lagrange multipliers.

For linear programming problems in $L^{p}$-spaces with pointwise constraints of bottleneck-type it has been known for a long time that associated Lagrange multipliers may exist in $L^{p}$-spaces, too. We mention for instance Grinold [7],[8], Levinson [10], and Tyndall [14]. Moreover, we refer to Anderson [1] and the monography by Anderson and Nash [2].

Bottleneck problems appear in optimal control problems for ODEs with certain mixed control-state inequality constraints. However, it seems that this kind of problems has not been studied since many years; nevertheless we may refer to a paper by Anderson and Philpott [3] which deals with

Maïtine Bergounioux, Dépt. de Mathématiques, UMR 6628, Université d'Orléans, 45067 Orléans, France. E-mail: Maitine.Bergouni oux@labomath.univ-orleans.fr.

Fredi Tröltzsch, Technical University of Chemnitz, Faculty of Mathematics, D-09107 Chemnitz, Germany. E-mail: f.troeltzsch@mathematik.tu-chemnitz.de.

This work was supported by EEC, HCM Contract CHRX-CT94-0471.

Received by the journal September 23, 1997. Revised April 7, 1998. Accepted for publication May 6, 1998.

(C) Société de Mathématiques Appliquées et Industrielles. Typeset by LATEX. 
a ODE problem close to the PDE problem we are going to investigate. Indeed, the associated theory can be extended also to the control of PDEs, see Bergounioux and Tiba [4] and the short note of Tröltzsch [13]. Here, we aim to extend those ideas to make them applicable to state-constrained control problems governed by PDEs instead of ODEs.

In contrast to the results on linear continuous programming, where the corresponding integral operators have bounded and measurable kernels, we shall have to deal with weakly singular operators. Moreover, we have to consider Lebesgue spaces $L^{p}(Q), 1<p<\infty$, to get the maximum regularity for Lagrange multipliers. In the early papers we mentioned above, only spaces of $L^{\infty}$-type appeared. To our knowledge, bottleneck problems have not been studied yet from this extended point of view.

We develop the theory for linear problems with application to a linear parabolic optimal distributed control problem as an example. In a forthcoming paper, a class of nonlinear parabolic control problems with pointwise mixed control-state inequality constraints will be discussed using these results.

In this way we hope to contribute with a class of problems, where different methods such as augmented Lagrangian methods, Lagrange-Newton methods, or second-order sufficient optimality conditions can be justified by having sufficiently regular multipliers in $L^{p}$-spaces.

The paper is organized as follows: first we introduce a class of linear programming problems in $L^{\infty}(Q)$ where $Q \subset \mathbb{R}^{N+1}$ is a certain measurable set. On extending the problem to $L^{p}(Q)(p>N / 2+1)$ (without changing the feasible set, which remains a subset of $L^{\infty}(Q)$ ), the associated dual problem is defined. In sections 4 and 5 the existence of solutions to the dual problems is shown for different choices of primal problems. In section 6 we investigate duality relations. Finally, linear control problems for PDEs are discussed in section 7 and 8 .

We consider the so-called Primal Problem

$$
(\mathcal{P})\left\{\begin{array}{l}
\max \int_{Q} a(x, t) u(x, t) d x d t \\
c_{1}(x, t) \leq u(x, t)-x \int_{0}^{t} \int_{\Omega} G(x, \xi, t, s) u(\xi, s) d \xi d s \leq c_{2}(x, t) \\
d_{1}(x, t) \leq u(x, t) \leq d_{2}(x, t) \text { a.e. in } Q .
\end{array}\right.
$$

In this setting, a bounded, measurable subset $\Omega \subset \mathbb{R}^{N}, N \geq 1, Q:=\Omega \times$ $(0, T)$ and functions $a \in L^{\infty}(Q), c_{i}, d_{i} \in L^{\infty}(Q), i=1,2$, are given, where $T>0$ is fixed. Define $D=\left\{(t, s) \in \mathbb{R}^{2} \mid 0 \leq s<t \leq T\right\}$ and a continuous function $G: \Omega^{2} \times D \rightarrow \mathbb{R}$ supposed to satisfy

$$
|G(x, \xi, t, s)| \leq k_{1}(t-s)^{-\frac{N}{2}} \exp \left(-k_{2} \frac{|x-\xi|^{2}}{t-s}\right),
$$

where $k_{1}, k_{2}$ are positive real numbers (we have in mind Green's functions $G$ for parabolic partial differential equations).

The main part of our analysis is devoted to the case $c_{1}=-\infty, c_{2}<\infty$, $d_{1}=0, d_{2}=\infty$, and to $c_{1}=-\infty, c_{2}<\infty, d_{1}=0, d_{2}<\infty$. The cases $c_{1}=$ $-\infty, c_{2}=+\infty$ and $d_{1}=-\infty, d_{2}=+\infty$ are more or less trivial. However, ESAIM: COCV, JunE 1998, Vol. 3, 235-250 
we were not able to show the existence of regular Lagrange multipliers for the general case of $(\mathcal{P})$, where all bounds really appear.

\section{AN INTEGRAL EQUATION}

We would like to discuss first the integral equation

$$
u(x, t)=c(x, t)+\int_{0}^{t} \int_{\Omega} G(x, \xi, t, s) u(\xi, s) d \xi d s
$$

for given $c \in L^{\infty}(Q)$. Equations of this type are basic for our theory. Let us introduce the integral operator $K$ :

$$
(K u)(x, t)=\int_{0}^{t} \int_{\Omega} G(x, \xi, t, s) u(\xi, s) d \xi d s .
$$

It is easy to show that $K$ is continuous in $L^{\infty}(Q)$, and from $L^{p}(Q)$ to $L^{\infty}(Q)$ for $N \geq 2, p>N / 2+1$ and $N=1, p>2$. We refer to Tröltzsch [11], p. 138, Lemma 5.6.6.

For convenience, we endow the space $L^{\infty}(Q)$ with the equivalent norm $\|u\|_{\lambda}$,

$$
\|u\|_{\lambda}=\sup _{(x, t) \in Q} \operatorname{ess}\left|e^{-\lambda t} u(x, t)\right|,
$$

where $\lambda>0$ (it holds $\left.e^{-\lambda T}\|u\|_{L^{\infty}(Q)} \leq\|u\|_{\lambda} \leq\|u\|_{L^{\infty}(Q)}\right)$.

LEMMA 2.1. $K$ is a contraction in $L^{\infty}(Q)$, for sufficiently large $\lambda>\lambda_{0}$.

Proof. In view of the definition of $\|u\|_{\lambda}$ and the estimate (1.1) we get

$$
\begin{aligned}
\|K u\|_{\lambda} & =\operatorname{supess}_{(x, t) \in Q}\left|e^{-\lambda t} \int_{0}^{t} \int_{\Omega} G(x, \xi, t, s) e^{\lambda s} e^{-\lambda s} u(\xi, s) d \xi d s\right| \\
& \leq \operatorname{supess}_{(x, t) \in Q} \int_{0}^{t} \int_{\mathbb{R}^{N}} k_{1}(t-s)^{-\frac{N}{2}} e^{-\lambda(t-s)} \\
& \exp \left(-k_{2} \frac{|x-\xi|^{2}}{t-s}\right) d \xi d s\|u\|_{\lambda} \\
& =\sup _{(x, t) \in Q} \operatorname{ess} k \int_{0}^{t} e^{-\lambda(t-s)} d s\|u\|_{\lambda} \leq \frac{k}{\lambda}\|u\|_{\lambda}
\end{aligned}
$$

where $k=k_{1} k_{2}^{-\frac{N}{2}} \pi^{\frac{N}{2}}$ (note that $\int_{-\infty}^{\infty} e^{-k \xi^{2}} d \xi=\sqrt{\pi / k}, k>0$ ).

Corollary 2.2. For all $c \in L^{\infty}(Q)$, the equation (2.1) has a unique solution $u \in L^{\infty}(Q)$. The mapping $c \mapsto u$ is continuous in $L^{\infty}(Q)$.

Proof. We have

$$
u=(I-K)^{-1} c=\left(\sum_{n=0}^{\infty} K^{n}\right) c
$$

by well known results on Neumann series. Moreover, $\left\|(I-K)^{-1}\right\|_{\lambda} \leq 1 /(1-$ $\left.\|K\|_{\lambda}\right)$, where we have used for convenience the symbol $\|\cdot\|_{\lambda}$ to denote also the norm of the operator $K$ induced by $\|\cdot\|_{\lambda}$. The result follows from the equivalence of the norms $\|\cdot\|_{\lambda}$ and $\|\cdot\|_{L^{\infty}(Q)}$. 
Corollary 2.3. If $G(x, \xi, t, s) \geq 0$ on $\Omega^{2} \times D$ and $c(x, t) \geq 0$ a.e. in $Q$, $c \in L^{\infty}(Q)$, then $u(x, t) \geq 0$ a.e. in $Q$.

Proof. The Neumann series representation (2.4) yields this result, as $c \geq 0$ implies $K^{n} c \geq 0 \forall n \in \mathbb{N}$.

Corollary 2.4. (Comparison principle). Suppose that $G(x, \xi, t, s) \geq 0$ on $\Omega^{2} \times D$ and $u_{i} \in L^{\infty}(Q), i=1,2$, are solutions of (2.1) associated to $c_{i} \in L^{\infty}(Q), i=1,2$. If $c_{1}(x, t) \geq c_{2}(x, t)$ holds a.e. in $Q$, then $u_{1}(x, t) \geq$ $u_{2}(x, t)$ a.e. in $Q$.

This result follows immediately from Corollary 2.3 .

In the next corollary we consider $K$ as an operator from $L^{p}(Q)$ to $L^{\infty}(Q)$. Corollary 2.5. (Extended comparison principle). Suppose $p \geq N / 2+1$. Then the integral equation (2.1) has for each $c \in L^{p}(Q)$ a unique solution $u \in L^{p}(Q)$, and the mapping $c \mapsto u$ is continuous in $L^{p}(Q)$.

Assume $G(x, \xi, t, s) \geq 0$ on $\Omega^{2} \times D, c_{i} \in L^{p}(Q), i=1,2, c_{1}(x, t) \geq c_{2}(x, t)$ a.e.in $Q$ and let $u_{i} \in L^{p}(Q), i=1,2$, be the associated solutions of (2.1). Then $u_{1}(x, t) \geq u_{2}(x, t)$ a.e. in $Q$.

Proof. We put $v:=u-c$, then $(2.1)$ reads

$$
v=K c+K v \text {. }
$$

As $K: L^{p}(Q) \rightarrow L^{\infty}(Q)$, continuously, the right hand side $K c$ belongs to $L^{\infty}(Q)$ and depends continuously on $c$. By Corollary 2.2, equation (2.5) admits a unique solution in $v \in L^{\infty}(Q)$ depending continuously on $K c$ and hence on $c$. Clearly, $u=c+v$ is a solution of $(2.1)$ in $L^{p}(Q)$.

The uniqueness of $u$ in $L^{p}(Q)$ is seen as follows: If $u_{1}, u_{2}$ are solutions in $L^{p}(Q)$ associated to the same $c$, then $u=u_{1}-u_{2}$ satisfies $u=K u$. Since $u \in L^{p}(Q)$, the smoothing property of $K$ implies even $u \in L^{\infty}(Q)$. Uniqueness in $L^{\infty}(Q)$ yields $u=0$. Therefore, $u_{1}=u_{2}$ must hold in $L^{p}(Q)$.

Finally, we derive the comparison principle assuming $G \geq 0$. Consider once again the auxiliary function $v:=u-c$. If $c \geq 0$, then $K c \geq 0$, too. From equation (2.5) and the first comparison principle we get $v \geq 0$ a.e. on Q. Therefore,

$$
u=c+v \geq c \geq 0
$$

holds true. The comparison principle is an easy consequence (put $c:=$ $\left.c_{1}-c_{2}\right)$.

So far, we have considered the integral operator $K$. This operator will appear in our primal problem. Our theory is focused on the associated dual problem, where the (formal) adjoint operator $K^{\top}$

$$
\left(K^{\top} \mu\right)(x, t)=\int_{t}^{T} \int_{\Omega} G(\xi, x, s, t) \mu(\xi, s) d \xi d s
$$

plays a crucial role. $K^{\top}$ is positive and has the same singularity as $K$. Therefore, $K^{\top}$ behaves like $K$. In particular, it is a contraction for $\lambda>\lambda_{0}$ in the norm $\|\cdot\|_{\lambda}$. It will be useful to prove the following result:

ESAIM: Cocv, June 1998, Vol. 3, 235-250 
Lemma 2.6. The equation

$$
\mu(x, t)=\max \left\{0, a(x, t)+\int_{t}^{T} \int_{\Omega} G(\xi, x, s, t) \mu(\xi, s) d \xi d s\right\}
$$

has exactly one solution $\mu \in L^{\infty}(Q)$.

Proof. Define by $\Pi$ the projection on the positive cone of $L^{\infty}(Q)$,

$$
(\Pi z)(x, t)=\max \{0, z(x, t)\}=: z(x, t)^{+} .
$$

Obviously

$$
\left|\left(\Pi z_{1}-\Pi z_{2}\right)(x, t)\right| \leq\left|\left(z_{1}-z_{2}\right)(x, t)\right| \quad \text { a.e. in } Q
$$

therefore, we get

$$
\left\|\Pi z_{1}-\Pi z_{2}\right\|_{\lambda} \leq\left\|z_{1}-z_{2}\right\|_{\lambda}
$$

by multiplying with $e^{-\lambda t}$ and taking the supremum over $Q$. The operator $\mathcal{T}$, expressed by the right hand side of $(2.7)$,

$$
\mathcal{T} \mu=\left[a+K^{\top} \mu\right]^{+},
$$

is a contraction in $L^{\infty}(Q)$ endowed with $\|\cdot\|_{\lambda}$ for $\lambda$ large enough:

$$
\begin{aligned}
\left\|\mathcal{T} \mu_{1}-\mathcal{T} \mu_{2}\right\|_{\lambda} & =\left\|\Pi\left(a+K^{\top} \mu_{1}\right)-\Pi\left(a+K^{\top} \mu_{2}\right)\right\|_{\lambda} \\
& \leq\left\|K^{\top} \mu_{1}-K^{\top} \mu_{2}\right\|_{\lambda} \\
& <\frac{k}{\lambda}\left\|\mu_{1}-\mu_{2}\right\|_{\lambda},
\end{aligned}
$$

is a contraction for $\lambda>k$.

Equation (2.7) reads $\mu=\mathcal{T} \mu$. According to the Banach fixed point theorem, this equation has a unique solution in $L^{\infty}(Q)$.

\section{TWO BASIC PRIMAL PROBLEMS}

From now, we assume that $G(x, \xi, t, s) \geq 0$ on $\Omega^{2} \times D$ and $p \geq N / 2+1$. Let us discuss two main choices of $(\mathcal{P})$.

We consider the problems $\left(\mathcal{P}_{1}\right),\left(\mathcal{P}_{2}\right)$,

$$
\begin{aligned}
& \left(\mathcal{P}_{1}\right) \begin{cases}\max \int_{Q} a(x, t) u(x, t) d x d t & \\
u(x, t) \leq[c+K u](x, t) & \text { a.e. in } Q, \\
u(x, t) \geq 0 & \text { a.e. in } Q,\end{cases} \\
& \left(\mathcal{P}_{2}\right) \begin{cases}\max \int_{Q} a(x, t) u(x, t) d x d t & \text { a.e. in } Q, \\
u(x, t) \leq[c+K u](x, t) & \text { a.e. in } Q . \\
0 \leq u(x, t) \leq d(x, t) & \end{cases}
\end{aligned}
$$

where $a, c$ and $d$ are $L^{\infty}(Q)$ functions.

We start with $\left(\mathcal{P}_{1}\right)$. Let us define $u_{c}$ by

$$
u_{c}=c+K u_{c} \text {. }
$$

Note that $u_{c}$ is bounded and measurable according to Corollary 2.2.

Theorem 3.1. $\left(\mathcal{P}_{1}\right)$ has a solution $\bar{u}$ if and only if $u_{c} \geq 0$. 
Proof. If $u_{c} \geq 0$, then the feasible domain for $\left(\mathcal{P}_{1}\right)$ is non empty. All feasible elements $u \in L^{p}(Q)$ satisfy $u \leq u_{c}$ by the second comparison principle of corollary 2.5, and we know $u_{c} \in L^{\infty}(Q)$. Moreover, $u \geq 0$ follows from the constraints, too. This implies the $L^{\infty}$-boundedness of $u$ by $\left\|u_{c}\right\|_{L^{\infty}(Q)}$. So the feasible set is bounded, closed and convex in all (reflexive) $L^{p}$-spaces for $1+N / 2<p<\infty$. The existence of $\bar{u} \in L^{p}(Q)$ is an immediate conclusion. Of course, $\bar{u}$ belongs to $L^{\infty}(Q)$.

On the other hand, if $u_{c}$ is not equal or greater than 0 , then the feasible set is empty. This follows again from the comparison principle.

THEOREM 3.2. If $u_{c} \geq 0$ (that is, if the feasible set is non empty) and if $d \geq 0$, then $\left(\mathcal{P}_{2}\right)$ has a solution.

The result is shown in the same way as for the preceding one.

\section{Dual Problem to $\left(\mathcal{P}_{1}\right)$}

Let us extend the feasible set of $\left(\mathcal{P}_{1}\right)$ from $L^{\infty}(Q)$ to $L^{p}(Q)(p>N / 2+1)$. Any feasible solution $u$ of $\left(\mathcal{P}_{1}\right)$ satisfies $0 \leq u \leq u_{c}$, where $u_{c}$ is defined in (3.1). Therefore, all feasible solutions of $L^{p}(\bar{Q})$ belong automatically to $L^{\infty}(Q)$, and the feasible set is independent of this extension to $L^{p}(Q)$.

Using standard techniques for constructing dual problems (cf. our discussion in section 6 ), we consider $\left(\mathcal{P}_{1}\right)$ in $L^{p}(Q)$ and get the following dual problem in $L^{q}(Q)$.

$$
\left(\mathcal{D}_{1}\right) \begin{cases}\min \int_{Q} c(x, t) \mu(x, t) d x d t & \\ \mu(x, t) \geq\left[a+K^{\top} \mu\right](x, t) & \text { a.e. in } Q \\ \mu(x, t) \geq 0 & \text { a.e. in } Q\end{cases}
$$

where $\mu \in L^{q}(Q), p^{-1}+q^{-1}=1$, and $K^{\top}$ is the integral operator defined by $(2.6)$.

The kernel of $K^{\top}$ satisfies the estimate (1.1), hence $K^{\top}: L^{p}(Q) \rightarrow L^{\infty}(Q)$ for $p>N / 2+1$, too. However, this is not true from $L^{q}(Q)$ to $L^{\infty}(Q)$. On the other hand, $K^{\top}$ represents the adjoint operator of $K: L^{p}(Q) \rightarrow L^{\infty}(Q) \subset$ $L^{p}(Q)$. Therefore, $K^{\top}$ is continuous in $L^{q}(Q) \sim L^{p}(Q)^{*}$, as well. In view of (1.1) and Lemma 2.1, $K^{\top}$ is a contraction in $L^{\infty}(Q)$, hence the equation

$$
\mu=\beta+K^{\top} \mu
$$

has for each $\beta \in L^{\infty}(Q)$ a unique solution $\mu \in L^{\infty}(Q)$. Moreover, we have uniqueness for (4.1) in $L^{q}(Q)$. Suppose that $\mu \in L^{q}(Q)$ solves the homogeneous equation $\mu-K^{\top} \mu=0$; we take an arbitrary $v \in L^{p}(Q)$, then

$$
0=\int_{Q} v\left(\mu-K^{\top} \mu\right) d x d t=\int_{Q} \mu(v-K v) d x d t .
$$

In view of the proof of Corollary 2.5, the range of $v-K v$ is $L^{p}(Q)$, hence $\mu=0$. This yields uniqueness for (4.1) in $L^{q}(Q)$.

REMARK 4.1. It is easy to see that the solution $\mu^{*}$ of

$$
\mu(x, t)=|a(x, t)|+\left(K^{\top} \mu\right)(x, t) \quad \text { a.e. in } Q,
$$


is a feasible and essentially bounded solution of $\left(\mathcal{D}_{1}\right)$ (this follows from the corollaries of section 2.)

THEOREM 4.2. If $c \geq 0$, then $\left(\mathcal{D}_{1}\right)$ has at least one optimal solution $\bar{\mu}$ given by equation (2.7).

Proof. Let $\bar{\mu}$ be the unique solution of (2.7). Suppose that $\mu \in L^{q}(Q)$ is any other feasible element for $\left(\mathcal{D}_{1}\right)$ (different from $\bar{\mu}$ ). Then

$$
\mu \geq a+K^{\top} \mu \text { a.e. in } Q
$$

and, of course, $\mu \geq 0$. Next, we construct a sequence $\mu_{1} \geq \mu_{2} \geq \cdots$ as follows: we put $\mu_{1}=\mu \in L^{q}(Q)$ and define

$$
\mu_{2}=\max \left\{0, a+K^{\top} \mu_{1}\right\} .
$$

A simple discussion yields $\mu_{2} \leq \mu_{1}$ a.e. in $Q$. Then the positivity of $K^{\top}$ implies $K^{\top} \mu_{2} \leq K^{\top} \mu_{1}$, and we get

$$
\mu_{2} \geq a+K^{\top} \mu_{1} \geq a+K^{\top} \mu_{2} \text { a.e. on } Q \text {. }
$$

Thus $\mu_{2}$ is feasible, and $\mu_{2} \leq \mu_{1}$ holds on $Q$. Repeating this process, one constructs a non-increasing feasible sequence $\left\{\mu_{n}\right\}$, which has to be pointwisely convergent towards some $\tilde{\mu} \geq 0$, i.e.

$$
\lim _{n \rightarrow+\infty} \mu_{n}(x, t)=\tilde{\mu}(x, t) \text { a.e. on } Q \text {. }
$$

Therefore, $\left\{\mu_{n}(x, t)-\tilde{\mu}(x, t)\right\}^{q}$ tends pointwisely to zero, too. Moreover, this sequence is bounded by $\left(\mu_{1}(x, t)\right)^{q} \in L^{1}(Q)$. The Lebesgue dominated convergence theorem yields that $(\mu-\tilde{\mu})^{q}$ tends to zero in $L^{1}(Q)$, hence $\mu_{n}$ tends to $\tilde{\mu}$ in $L^{q}(Q)$.

$K^{\top}$ is continuous in $L^{q}(Q)$ so that $a+K^{\top} \mu_{n} \rightarrow a+K^{\top} \tilde{\mu}$ in $L^{q}(Q)$. Passing to the limit in

$$
\mu_{n}=\max \left\{0, a+K^{\top} \mu_{n-1}\right\}
$$

(the right hand side is a continuous mapping in $L^{q}(Q)$ ) gives

$$
\tilde{\mu}=\max \left\{0, a+K^{\top} \tilde{\mu}\right\} .
$$

We are able to conclude $\tilde{\mu} \in L^{\infty}(Q)$. This is seen from the equation

$$
\tilde{\mu}(x, t)=\beta(x, t) a(x, t)+\beta(x, t)\left(K^{\top} \tilde{\mu}\right)(x, t),
$$

where $\beta(x, t)=0$ for $\tilde{\mu}(x, t)=0$ and $\beta(x, t)=1$ for $\tilde{\mu}(x, t)>0$. This equation is of the type discussed in section 2, where $c=\beta a \in L^{\infty}(Q)$ (the new kernel $\widetilde{G}=\beta G$ satisfies the estimate (1.1) too and $\widetilde{G} \geq 0$.) Hence $\tilde{\mu} \in L^{\infty}(Q)$. By uniqueness in $L^{\infty}(Q)$ we have $\tilde{\mu}=\bar{\mu}$; moreover $\mu \geq \tilde{\mu}$.

Consequently $\mu \geq \bar{\mu}$ holds for all feasible solutions, and

$$
\int_{Q} c(x, t) \mu(x, t) d x d t \geq \int_{Q} c(x, t) \bar{\mu}(x, t) d x d t
$$

follows from $c \geq 0$. 


\section{Dual Problem to $\left(\mathcal{P}_{2}\right)$}

In the case of $\left(\mathcal{P}_{2}\right)$ we obtain the dual problem

$$
\left(\mathcal{D}_{2}\right) \begin{cases}\min \int_{Q}\left[c(x, t) \mu_{1}(x, t)+d(x, t) \mu_{2}(x, t)\right] d x d t & \\ \mu_{1}(x, t)+\mu_{2}(x, t) \geq\left[a+K^{\top} \mu_{1}\right](x, t) & \text { a.e. in } Q \\ \mu_{1}(x, t) \geq 0, \mu_{2}(x, t) \geq 0 & \text { a.e. in } Q\end{cases}
$$

where the unknown functions $\mu_{i}, i=1,2$ belong to $L^{q}(Q)$.

From now, we assume $c \geq 0$ and $d \geq 0$.

LEMMA 5.1. For any feasible pair of $\left(\mathcal{D}_{2}\right)$ having the objective functional value $v$, there exists another feasible pair $\left(\bar{\mu}_{1}, \bar{\mu}_{2}\right)$ such that

$$
\bar{\mu}_{1}+\bar{\mu}_{2}=\max \left\{0, a+K^{\top} \bar{\mu}_{1}\right\}
$$

and the associated value is not greater than $v$.

Proof. Let $\left(\mu_{1}, \mu_{2}\right)$ be a feasible pair with value $v$ for the objective functional. Then we know

$$
\mu_{1}+\mu_{2} \geq \max \left\{0, a+K^{\top} \mu_{1}\right\} .
$$

Once again, we define decreasing sequences $\left\{\mu_{1}^{n}\right\},\left\{\mu_{2}^{n}\right\}$ as follows:

$$
\begin{gathered}
\mu_{j}^{1}:=\mu_{j}, j=1,2, \\
\mu_{1}^{n+1}+\mu_{2}^{n+1}=\max \left\{0, a+K^{\top} \mu_{1}^{n}\right\}, n=1,2, \ldots
\end{gathered}
$$

More precisely, assume that $\mu_{1}^{n} \geq 0$ and $\mu_{2}^{n} \geq 0$ have been constructed as above and satisfy

$$
\mu_{1}^{n}+\mu_{2}^{n} \geq \max \left\{0, a+K^{\top} \mu_{1}^{n}\right\}=\nu^{n} \geq 0 .
$$

We set $\mu_{i}^{n+1}=\alpha^{n} \mu_{i}^{n}, i=1,2$, where

$$
\alpha^{n}(x, t)= \begin{cases}0 & \text { if } \nu^{n}(x, t)=0, \\ \frac{\nu^{n}(x, t)}{\mu_{1}^{n}(x, t)+\mu_{2}^{n}(x, t)} & \text { otherwise. }\end{cases}
$$

Thus $0 \leq \alpha^{n}(x, t) \leq 1$ and we have $0 \leq \mu_{i}^{n+1} \leq \mu_{i}^{n}$. The very choice of $\alpha^{n}$ gives $\mu_{i}^{n+1} \geq 0$ and $\mu_{1}^{n+1}+\mu_{2}^{n+1}=\nu^{n}=\max \left\{0, a+K^{\top} \mu_{1}^{n}\right\}$. The positivity of $K$ and $0 \leq \mu_{1}^{n+1} \leq \mu_{1}^{n}$ yield that $\max \left\{0, a+K^{\top} \mu_{1}^{n}\right\} \geq \max \left\{0, a+K^{\top} \mu_{1}^{n+1}\right\}$ and

$$
\mu_{1}^{n+1}+\mu_{2}^{n+1} \geq \max \left\{0, a+K^{\top} \mu_{1}^{n+1}\right\}=\nu^{n+1} \geq 0 .
$$

Passing to the limit along the lines of the last proof, we get

$$
\lim _{n \rightarrow \infty} \mu_{i}^{n}=\bar{\mu}_{i} \text { in the sense of } L^{q}(Q)
$$

and

$$
\begin{aligned}
\lim _{n \rightarrow \infty} \nu^{n} & =\lim _{n \rightarrow \infty} \max \left\{0, a+K^{\top} \mu_{1}^{n}\right\} \\
& =\max \left\{0, a+K^{\top} \bar{\mu}_{1}\right\} \text { in the sense of } L^{q}(Q) .
\end{aligned}
$$

Moreover,

$$
\bar{\mu}_{1}+\bar{\mu}_{2}=\max \left\{0, a+K^{\top} \bar{\mu}_{1}\right\} .
$$

Clearly, $\bar{v} \leq v$ holds for the value of the objective functional as $c \geq 0$ and $d \geq 0$. 
So far, we have shown comparison principles in $L^{p}(Q), p>N / 2+1$. Let us now add a third conclusion for $L^{q}(Q)$.

LEMMA 5.2. If $\alpha \geq 0$ is given in $L^{q}(Q)$ and $\mu \in L^{q}(Q)$ is the solution of

$$
\mu=\alpha+K^{\top} \mu
$$

then $\mu \geq 0$ holds a.e. on $Q$.

Proof. This is shown as follows: define

$$
\mathcal{M}^{-}=\{(x, t) \in Q \mid \mu(x, t)<0\}
$$

and

$$
\beta=\chi_{\mathcal{M}^{-}}= \begin{cases}1 & \text { if }(x, t) \in \mathcal{M}^{-}, \\ 0 & \text { otherwise. }\end{cases}
$$

Let $v \in L^{\infty}(Q)$ be the unique solution of $v-K v=\beta$. By the extended comparison principle we have $v \geq 0$. Now multiply (5.2) by $v$ and integrate over $Q$. Then

$$
\begin{aligned}
\int_{Q} \alpha(x, t) v(x, t) d x d t & =\int_{Q}\left(\mu(x, t)-K^{\top} \mu(x, t)\right) v(x, t) d x d t \\
& =\int_{Q}(v(x, t)-K v(x, t)) \mu(x, t) d x d t \\
& =\int_{\mathcal{M}^{-}} \mu(x, t) d x d t .
\end{aligned}
$$

From $\alpha, v \geq 0$ we conclude that $\left(\mathcal{M}^{-}\right)$has a null measure; hence $\mu \geq 0$ a.e. on $Q$.

Theorem 5.3. Problem $\left(\mathcal{D}_{2}\right)$ admits at least one solution $\left(\bar{\mu}_{1}, \bar{\mu}_{2}\right) \in L^{\infty}(Q)^{2}$.

Proof. Owing to Lemma 5.1 we may restrict ourselves to the set of all pairs $\left(\mu_{1}, \mu_{2}\right) \in L^{q}(Q)^{2}$ such that

$$
\mu_{1}+\mu_{2}=\max \left\{0, a+K^{\top} \mu_{1}\right\} \text { and } \mu_{j} \geq 0, j=1,2 .
$$

Therefore,

$$
\mu_{1} \leq \mu_{1}+\mu_{2} \leq|a|+K^{\top} \mu_{1} .
$$

So we have $\mu_{1} \leq|a|+K^{\top} \mu_{1}$. Consider the solution $\mu_{a}$ of

$$
\mu_{a}(x, t)=|a(x, t)|+\left(K^{\top} \mu_{a}\right)(x, t) \quad \text { a.e. in } Q \text {. }
$$

This solution belongs to $L^{\infty}(Q)$ (applying Lemma 2.1 to $K^{\top}$ as an operator from $L^{p}(Q)$ to $L^{\infty}(Q)$ ) and is unique in $L^{q}(Q)$ ( see the discussion of (4.1)). We have

$$
\mu_{1}=\alpha+K^{\top} \mu_{1}
$$

where $\alpha(x, t) \leq|a(x, t)|$ and $\alpha \in L^{q}(Q)$. The comparison principle of Lemma 5.2 yields $\mu_{1} \leq \mu_{a}$, hence $0 \leq \mu_{1}$ implies $\mu_{1} \in L^{\infty}(Q)$.

Therefore $\mu_{2}$ is uniformly bounded as well. In this way we may restrict to a weakly compact subset of $L^{q}(Q)$, which is uniformly bounded. We complete the proof by standard arguments. 
We are able to check the existence of solutions to the dual problem $(\mathcal{D})$ for some other meaningful choices of the constraints of $(\mathcal{P})$, too; consider the problem

$$
\left\{\begin{array}{l}
\max \int_{Q} a u d x d t \\
d_{1} \leq u \leq d_{2}
\end{array}\right.
$$

This case is obtained for $c_{1}=-\infty, c_{2}=\infty$. The dual is

$$
\left\{\begin{array}{l}
\min \int_{Q}\left(d_{2} \mu_{2}-d_{1} \mu_{1}\right) d x d t \\
\mu_{2}-\mu_{1}=a \\
\mu_{i} \geq 0, i=1,2
\end{array}\right.
$$

An easy exercise gives the solution $\mu_{1}=a^{-}, \mu_{2}=a^{+}$for $\left(\mathcal{D}_{3}\right)$. The choice $d_{1}=-\infty, d_{2}=\infty$ defines

$$
\left\{\begin{array}{l}
\max \int_{Q} a u d x d t \\
c_{1} \leq u-K u \leq c_{2}
\end{array}\right.
$$

having the dual problem

$$
\left\{\begin{array}{l}
\min \int_{Q}\left(d_{2} \mu_{2}-d_{1} \mu_{1}\right) d x d t \\
(I-K)\left(\mu_{2}-\mu_{1}\right)=a \\
\mu_{i} \geq 0, i=1,2
\end{array}\right.
$$

Problem $\left(\mathcal{P}_{4}\right)$ is transformed on substituting $v=u-K u$ to one of the type $\left(\mathcal{P}_{3}\right)$. We obtain $u=R v$ with $R=\sum_{n=0}^{\infty} K^{n}$. The transformed problem is

$$
\max \int_{Q} \tilde{a} v d x d t, \quad c_{1} \leq v \leq c_{2},
$$

with $\tilde{a}=R^{\top} a$. The solution $\mu_{1}=\tilde{a}^{-}, \mu_{2}=\tilde{a}^{+}$of the associated dual problem is easily seen to solve $\left(\mathcal{D}_{4}\right)$, too.

\section{The DUAlity Relation}

The discussion of the duality between $(\mathcal{P})$ and $(\mathcal{D})$ is not yet complete. We have only shown that under certain assumptions the dual problem admits a solution $\bar{\mu}$. To ensure that $\bar{\mu}$ is a Lagrange multiplier associated to a solution of $(\mathcal{P})$, we need additionally the strong duality relation, that is the equality of primal and dual optimal value. We shall prove that this relation holds true in all of our cases of interest. To this aim, we briefly sketch some main ideas of duality for linear programs, which are known from the papers [13], [14] and the general expositions in [1], [2].

Let $X$ and $Y$ be two real Banach spaces with nonempty convex closed cones $K_{X} \subset X$ and $K_{Y} \subset Y$. Let us denote by $X^{\prime}, Y^{\prime}$ the associated dual spaces. In $X$ we introduce a partial ordering $\geq_{X}$ by $x \geq_{X} 0$ if and only if $x \in K_{X}$. Analogously, $\geq_{Y}$ is defined in $Y$. In what follows, we shall freely use $\langle\cdot, \cdot\rangle$ to denote the pairing between $X$ and $X^{\prime}$ or $Y$ and $Y^{\prime}$. The dual cone $K_{X}^{+}$is defined by

$$
K_{X}^{+}=\left\{w \in X^{\prime} \mid\langle w, x\rangle \geq 0 \quad \forall x: x \geq_{X} 0\right\},
$$


and $K_{Y}^{+}$is introduced analogously in $Y^{\prime}$. In $X^{\prime}, Y^{\prime}$, partial orderings are defined by these cones $K_{X}^{+}$and $K_{Y}^{+}$. Finally, let fixed elements $a \in X^{\prime}$, $c \in Y$, and a linear continuous operator $A: X \rightarrow Y$ with adjoint $A^{\prime}$ be given.

The abstract primal problem is

$$
\left\{\begin{array}{l}
\max \langle a, x\rangle \\
A x \leq_{Y} c \\
x \geq_{X} 0
\end{array}\right.
$$

In what follows, we often omit the indexed space in the partial ordering, since the meaning of the ordering is clear from the context. On using the Lagrange function $\mathcal{L}: X \times Y^{\prime} \rightarrow \mathbb{R}$

$$
\mathcal{L}(x, \mu):=\langle a, x\rangle+\langle\mu, c-A x\rangle,
$$

the primal problem can be expressed in the form to find the value

$$
v=\sup _{x \geq 0} \inf _{\mu \geq 0} \mathcal{L}(x, \mu) .
$$

The dual problem is obtained in $Y^{\prime}$ by Lagrange duality, that is by reversing the order of sup and inf:

$$
v^{\prime}=\inf _{\mu \geq 0} \sup _{x \geq 0} \mathcal{L}(x, \mu)=\inf _{\mu \geq 0}\left\{\langle\mu, c\rangle+\sup _{x \geq 0}\left\langle a-A^{\prime} \mu, x\right\rangle\right\} .
$$

The last supremum is zero, if $a-A^{\prime} \mu \leq 0$, otherwise $+\infty$. Hence $(\mathcal{D})$ is nothing else than

$$
\left\{\begin{array}{l}
\min \langle\mu, c\rangle \\
A^{\prime} \mu \geq_{X^{\prime}} a \\
\mu \geq_{Y^{\prime}} 0
\end{array}\right.
$$

Let $\bar{x}$ be optimal for $(\mathcal{P})$. Then $\bar{\mu}$ is an associated Lagrange multiplier if and only if the pair $(\bar{x}, \bar{\mu})$ is a saddle point of $\mathcal{L}$,

$$
\mathcal{L}(x, \bar{\mu}) \leq \mathcal{L}(\bar{x}, \bar{\mu}) \leq \mathcal{L}(\bar{x}, \mu)
$$

for all $x \geq 0, \mu \geq 0$. A necessary and sufficient condition for $(\bar{x}, \bar{\mu})$ to be a saddle point is that $\bar{x}$ solves $(\mathcal{P}), \bar{\mu}$ solves $(\mathcal{D})$, and the strong duality relation $v=v^{\prime}$ holds true.

Let us define by $P(c)$ and $D(a)$ the feasible sets of $(\mathcal{P})$ and $(\mathcal{D})$, respectively, that is

$$
\begin{aligned}
P(c) & =\{x \in X \mid x \geq 0, A x \leq c\} \\
D(a) & =\left\{\mu \in Y^{\prime} \mid \mu \geq 0, A^{\prime} \mu \geq a\right\} .
\end{aligned}
$$

It is easy to check that the weak duality relation

$$
\sup _{x \in P(c)}\langle a, x\rangle \leq \inf _{\mu \in D(a)}\langle\mu, c\rangle
$$

always holds true. To show the strong duality relation we need the convex cone

$$
K(A):=\{(\alpha, d) \in \mathbb{R} \times Y \mid \exists x \geq 0: A x \leq d,\langle a, x\rangle \geq \alpha\}
$$


THEOREM 6.1. If $K(A)$ is closed and $(\mathcal{P})$ admits at least one solution, then the strong duality relation

$$
\max _{x \in P(c)}\langle a, x\rangle=\inf _{\mu \in D(a)}\langle\mu, c\rangle
$$

is true.

This is a standard result. We refer, for example, to [6] (the result is given for the general convex case). It turns out that the assumptions of the theorem are fulfilled in all of our cases of interest for $(\mathcal{P})$. Let us consider at first our most general primal problem

$$
\left\{\begin{array}{l}
\max \langle a, u\rangle \\
c_{1} \leq u-K u \leq c_{2} \\
0 \leq u \leq d
\end{array}\right.
$$

introduced in section 1 , where $\langle a, u\rangle=\int_{Q} a(x, t) u(x, t) d x d t$. Written in canonical form, the constraints are

$$
\begin{aligned}
u-K u & \leq c_{2} \\
-u+K u & \leq-c_{1} \\
u & \leq d \\
u & \geq 0 .
\end{aligned}
$$

Moreover, we have $X=L^{p}(Q), Y=\left(L^{p}(Q)\right)^{3}$ endowed with their natural partial ordering, and

$$
A=\left(\begin{array}{c}
I-K \\
-I+K \\
I
\end{array}\right)
$$

Lemma 6.2. $K(A)$ is closed for the choice of $(\mathcal{P})$ defined above.

Proof. In the abstract setting of $(\mathcal{P})$ we have $c=\left(c_{2},-c_{1}, d\right)^{\top}$. Let $\left(\alpha^{n}, c^{n}\right) \in$ $K(A)$ be given such that $\left(\alpha^{n}, c^{n}\right) \rightarrow(\alpha, c)$ in $\mathbb{R} \times Y$ as $n \rightarrow \infty$. Then there is a sequence $\left\{u^{n}\right\}$ such that

$$
A u^{n} \leq c^{n}, \quad u^{n} \geq 0, \quad\left\langle a, u^{n}\right\rangle \geq \alpha^{n} .
$$

This sequence is bounded. Here, this follows from $0 \leq u^{n} \leq d^{n}$. Moreover, $X$ is reflexive. Therefore, we may select a weakly converging subsequence and can assume that $u^{n} \rightarrow \hat{u}$. Convex closed cones are weakly closed, hence

$$
A \hat{u} \leq c, \quad \hat{u} \geq 0, \quad\langle a, \hat{u}\rangle \geq \alpha,
$$

that is $(\alpha, c) \in K(A)$.

A study of the proof reveals that its main point is the boundedness of the sequence $\left\{u_{n}\right\}$. The same property holds true in the following cases of the constraints for $(\mathcal{P})$ :

(i) $u-K u \leq c_{2}, \quad u \geq 0$.

Here we apply the comparison principle and get $u \leq u_{c}$, where $u_{c}$ is defined as in section $3, u_{c}-K u_{c}=c_{2}$. Convergence of $c_{2}^{n}$ implies that of $u_{c_{n}}$, hence boundedness of $u_{n}$.

(ii) $c_{1} \leq u-K u, \quad u \leq d, \quad u \geq 0$.

Boundedness is trivially satisfied.

(iii) $c_{1} \leq u-K u \leq c_{2}$.

ESAIM: CoCV, JUne 1998, Vol. 3, 235-250 
This case does not instantly fit into the abstract scheme, as the constraint $u \geq 0$ is missing. If we define $K_{X}=X$, then we can add formally $u \geq_{X} 0$ without changing the constraints. Here, the solution $u$ is bounded by the two solutions of the equations $u-K u=c_{1}$ and $u-K u=c_{2}$ (comparison principle). Therefore, the boundedness needed in the proof is fulfilled, too. Summarizing, the duality relation holds true in all our cases of interest.

\section{A ClASS OF LINEAR PARABOLIC CONTROL PROBLEMS WITH BOTTLENECK CONSTRAINTS}

Consider the following linear parabolic control problem

$$
(\mathcal{P})\left\{\begin{aligned}
\max & \left(\int_{\Omega} a_{\Omega}(x) y(x, T) d x+\int_{Q} a_{Q}(x, t) y(x, t) d x d t\right. \\
& \left.+\int_{\Sigma} a_{\Sigma}(x, t) y(x, t) d \sigma d t+\int_{Q} a_{u}(x, t) u(x, t) d x d t\right) \\
\text { subject to } & y_{t}+\operatorname{div}(\mathcal{A} \nabla y)+\ell_{y} y=\ell_{u} u \text { in } Q, \\
& \partial_{\nu} y+b_{y} y=0 \text { on } \Sigma, \\
& y(0)=0 \text { in } \Omega, \\
& 0 \leq u(x, t) \leq d(x, t) \text { in } Q, \\
& u(x, t) \leq c(x, t)+y(x, t) \text { in } Q .
\end{aligned}\right.
$$

In this setting, $\Omega$ is an open bounded domain of $\mathbb{R}^{N}$ with boundary $\Gamma$ and $T$ is a nonnegative real number. We set $Q=\Omega \times] 0, T[; \Sigma=\Gamma \times] 0, T[$ is the lateral boundary of $Q$ and $\partial_{\nu} y$ denotes the outward normal derivative on $\Gamma$. The (data) functions $a_{\Omega}, a_{Q}, a_{\Sigma}, a_{u}, \ell_{y}, \ell_{u}, b_{y}, d$, and $c$ are bounded and measurable on their domains. We assume $\ell_{y}, \ell_{u}, b_{y} \geq 0$. In addition, $\Gamma$ and the coefficients of the (symmetric uniformly negative definite) matrix $\mathcal{A}$ are supposed sufficiently smooth such that the associated Green's function $\widetilde{G}$ exists. Then the weak solution of the PDE is given by

$$
y(x, t)=\int_{0}^{t} \int_{\Omega} \widetilde{G}(x, \xi, t, s) \ell_{u}(\xi, s) u(\xi, s) d \xi d s=[K u](x, t) .
$$

$\widetilde{G}$ is known to be nonnegative in view of the maximum principle and satisfies (1.1). The objective admits the form

$$
\int_{0}^{T} \int_{\Omega} a(x, t) u(x, t) d x d t
$$

where

$$
\begin{aligned}
a(x, t)= & \left(\int_{\Omega} \widetilde{G}(x, \xi, t, s) a_{\Omega}(\xi, s) d \xi+\int_{t}^{T} \int_{\Omega} \widetilde{G}(x, \xi, t, s) a_{Q}(\xi, s) d \xi d s\right. \\
& \left.+\int_{t}^{T} \int_{\Gamma} \widetilde{G}(x, \xi, t, s) a_{\Sigma}(\xi, s) d \sigma d s\right) \ell_{u}(x, t)+a_{u}(x, t) .
\end{aligned}
$$

This follows from the Fubini theorem. On using the integral operator $K$ defined in section 2 , the constraints read

$$
u \leq c+K u, \quad 0 \leq u \leq d .
$$


Therefore the above problem $(\mathcal{P})$ fits in $\left(\mathcal{P}_{2}\right)$ defined in Section 3 . According to $\left(\mathcal{D}_{2}\right)$, the corresponding dual problem has the constraints $\mu_{1} \geq 0, \mu_{2} \geq$ $0, \mu_{1}+\mu_{2} \geq a+K^{\top} \mu_{1}$. Our theory allows to consider $\left(\mathcal{D}_{2}\right)$ in $L^{\infty}(Q)^{2}$. The function

$$
\varphi(x, t)=\int_{t}^{T} \int_{\Omega} \widetilde{G}(\xi, x, s, t) \mu_{1}(\xi, s) d \xi d s=K^{\top} \mu_{1}
$$

solves the adjoint parabolic equation

$$
-\varphi_{t}+\operatorname{div}(\mathcal{A} \nabla \varphi)+\ell_{y} \varphi=\mu_{1}
$$

in $Q$, subject to homogeneous boundary conditions and $\varphi(T)=0$. Substituting $\varphi$ for $K^{\top} \mu_{1}$, the dual problem admits the form of a dual optimal control problem with constraints of bottleneck type,

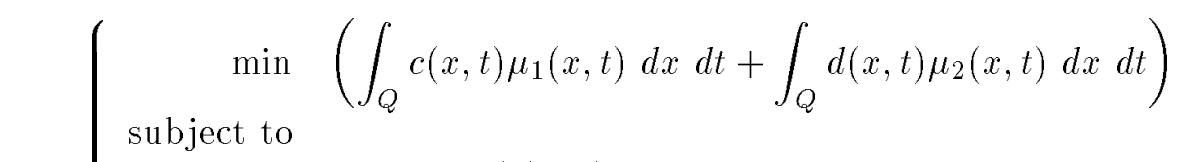

Invoking our theory we obtain the following result:

Theorem 7.1. For any solution $\bar{u}$ of $(\mathcal{P})\left(\right.$ in $L^{\infty}(Q)$ ), the dual problem has at least a solution $\left(\bar{\mu}_{1}, \bar{\mu}_{2}\right)$ which belongs to $L^{\infty}(Q)^{2}$. Moreover $\bar{\mu}_{i}, i=1,2$ are the Lagrange multipliers associated to $\bar{u}$.

\section{Extension to BOUNDARY CONTROL PROBLEMS}

We have concentrated so far on a distributed control problem aiming to simplify the presentations of the main ideas. Anyway, we may give some hints for a possible extension to the study of boundary control problems. Of course, the case we have presented in the previous section is a particular case of the one (roughly) described in this section. However, we decided to discuss first a simpler application to make the presentation clearer.

A study of our techniques immediately reveals that problems of the type

$$
(\mathcal{P})\left\{\begin{array}{c}
\max \left(\int_{Q} a_{1}(x, t) u(x, t) d x d t+\int_{\Sigma} a_{2}(x, t) v(x, t) d \sigma d t\right) \\
u(x, t) \leq c_{1}(x, t)+\int_{0}^{t} \int_{\Omega} G_{11}(x, \xi, t, s) u(\xi, s) d \xi d s \\
\quad+\int_{0}^{t} \int_{\Gamma} G_{12}(x, \xi, t, s) v(\xi, s) d \sigma d s \text { a.e. in } Q, \\
v(x, t) \leq c_{2}(x, t)+\int_{0}^{t} \int_{\Omega} G_{21}(x, \xi, t, s) u(\xi, s) d \xi d s \\
\quad+\int_{0}^{t} \int_{\Gamma} G_{22}(x, \xi, t, s) v(\xi, s) d \sigma d s \text { a.e. on } \Sigma, \\
0 \leq u(x, t) \leq d_{1}(x, t), \\
0 \leq v(x, t) \leq d_{2}(x, t),
\end{array}\right.
$$

can be treated analogously. Here $a_{1}, c_{1}, d_{1} \in L^{\infty}(Q), a_{2}, c_{2}, d_{2} \in L^{\infty}(\Sigma)$ and nonnegative kernels $G_{i j}: D \rightarrow \mathbb{R}$ are given satisfying the same type ESAIM: COCV, JUNE 1998, Vol. 3, 235-250 
of assumptions imposed on $G$ in section $1 . \Sigma=\Gamma \times(0, T)$ is the lateral boundary, and $d \sigma$ denotes the surface measure. We have to assume nonnegativity of $c_{i}, d_{i}, i=1,2$. The boundary integral operators defined by $G_{i 2}, i=1,2$, transform $L^{p}(\Sigma)$ into $L^{\infty}(Q)$ and $L^{\infty}(\Sigma)$ respectively provided that $p>N+1$, where $N=\operatorname{dim} \Omega$ (see Tröltzsch [11]). Hence the primal problem $(\mathcal{P})$ can be defined in $L^{p}(Q) \times L^{p}(\Sigma)$ for $p>N+1$. The associated operator $K$,

$$
K(u, v)(x, t)=\left(\begin{array}{l}
K_{11} u+K_{12} v \\
K_{21} u+K_{22} v
\end{array}\right)(x, t),
$$

is to be regarded as linear continuous operator in $L^{p}(Q) \times L^{p}(\Sigma)$. Its adjoint operator is defined in $L^{q}(Q) \times L^{q}(\Sigma)$ by

$$
\begin{aligned}
& K^{*}(\mu, z)(x, t)= \\
& \quad\left(\begin{array}{l}
\int_{t}^{T} \int_{\Omega} G_{11}(\xi, x, s, t) \mu(\xi, s) d \xi d s+\int_{t}^{T} \int_{\Gamma} G_{21}(\xi, x, s, t) z(\xi, s) d \sigma d s \\
\int_{t}^{T} \int_{\Omega} G_{12}(\xi, x, s, t) \mu(\xi, s) d \xi d s+\int_{t}^{T} \int_{\Gamma} G_{22}(\xi, x, s, t) z(\xi, s) d \sigma d s
\end{array}\right) .
\end{aligned}
$$

The symmetry of the exponential estimate (1.1) shows that

$$
K^{*}: L^{p}(Q) \times L^{p}(\Sigma) \rightarrow L^{\infty}(Q) \times L^{\infty}(\Sigma)
$$

continuously, for $p>N+1$ as well. The dual problem is obtained in $L^{q}(Q) \times L^{q}(\Sigma)$ by

$$
(\mathcal{D})\left\{\begin{aligned}
\min & \left(\int_{Q}\left(c_{1} \mu_{1}+d_{1} \mu_{2}\right) d x d t+\int_{\Sigma}\left(c_{2} z_{1}+d_{2} z_{2}\right) d \sigma d t\right) \\
\text { subject to } & \\
& \mu_{1}+\mu_{2} \geq a_{1}+K_{11}^{*} \mu_{1}+K_{21}^{*} z_{1}, \\
& z_{1}+z_{2} \geq a_{2}+K_{12}^{*} \mu_{1}+K_{22}^{*} z_{1}, \\
& \mu_{i} \geq 0, i=1,2, \\
& z_{i} \geq 0, i=1,2 .
\end{aligned}\right.
$$

Introducing an equivalent norm, $K$ and $K^{*}$ are seen to be contractions in $L^{\infty}$. Hence the theory of the preceding sections can be developed analogously.

We obtain optimal solutions of $(\mathcal{D})$ in $L^{\infty}(Q)^{2} \times L^{\infty}(\Sigma)^{2}$, if the functions $c_{i}, d_{i}, i=1,2$, are nonnegative. These results can be used to handle parabolic control problems of the type

$$
\left\{\begin{aligned}
\max & \left(\int_{\Omega} a_{\Omega} y(T) d x+\int_{Q} a_{Q} y d x d t+\int_{\Sigma} a_{\Sigma} y d \sigma d t\right. \\
& \left.+\int_{Q} a_{u} u d x d t+\int_{\Sigma} a_{v} v d \sigma d t\right) \\
\text { subject to } \quad & \\
& y_{t}+\operatorname{div}(\mathcal{A} \nabla y)+b_{1 y} y=b_{u} u \text { in } Q, \\
& \partial_{\nu} y+b_{2 y} y=b_{v} v \text { on } \Sigma, \\
& y(0)=y_{0} \text { in } \Omega, \\
& 0 \leq u(x, t) \leq d_{1} \text { in } Q, 0 \leq v(x, t) \leq d_{2} \text { on } \Sigma, \\
& u \leq c_{1}+y \text { in } Q, v(x, t) \leq c_{2}+y \text { on } \Sigma .
\end{aligned}\right.
$$

Here,

$$
\begin{aligned}
& G_{11}=G_{21}=b_{u}(\xi, s) G(x, \xi, t, s), \quad G_{21}=G_{22}=b_{v}(\xi, s) G(x, \xi, t, s) \\
& \text { ESAIM: CoCv, JUne } 1998 \text {, Vol. 3, 235-250 }
\end{aligned}
$$


where $G=G(x, \xi, t, s)$ is the Green's function associated to the parabolic initial-boundary value problem. $G$ satisfies the estimate (1.1) (see [11] and the references therein). The technique of our paper applies to show the existence of bounded and measurable Lagrange multipliers associated to the state constraints. We leave the details to the reader.

\section{REFERENCES}

[1] E.J. Anderson: A review of duality theory for linear programming over topological vector spaces, J. Math. Anal. Appl., 97, 1983, 380-392.

[2] E.J. Anderson, P. Nash: Linear programming in infinite-dimensional spaces, J. Wiley \& sons, Chichester, 1987.

[3] E.J. Anderson, A.B. Philpott: Extreme points for linear optimal control problems with diagonal structure, SIAM J. Control Optim., 30 (6), 1992, 1385-1394.

[4] M. Bergounioux, D. Tiba: General optimality conditions for constrained convex control problems, SIAM J. Control Optim., 34 (2), 1996, 698-711.

[5] M. Bergounioux, F. Tröltzsch: Optimality conditions and generalized Bang-Bang principle for a state constrained semilinear parabolic problem, Numerical Functional Analysis and Optimization, 15, 1996, 517-537.

[6] I. Ekeland, R. Temam: Analyse convexe et problèmes variationnels, Dunod GauthierVillars, Paris, 1974.

[7] R.C. Grinold: Continuous programming I, J. Math. Anal. Appl., 28, 1969, 39-51.

[8] R.C. Grinold: Symmetric duality for continuous linear programs, SIAM J. Appl. Math., 18, 1970, 84-97.

[9] A.D. Ioffe, V.M. Tikhomirov: Theory of extremal problems, North-Holland, Amsterdam, 1979 (translation from the Russian).

[10] N. Levinson: A class of continuous linear programming problems, J. Math. Anal. Appl., 16, 1966, 73-83.

[11] F. Tröltzsch: Optimality conditions for parabolic control problems and applications, Teubner Texte zur Mathematik, Leipzig, 1984.

[12] F. Tröltzsch: A modification of the Zowe and Kurcyusz regularity condition with application to the optimal control of Noether operator equations with constraints on the control and the state, Math. Operationforsch. Statist., Serie Optimization, 14 (2), $1983,245-253$.

[13] F. Tröltzsch: Existenz- und Dualitätsaussagen für lineare Optimierungsaufgaben in reflexiven Banachräumen, Math. Operationsforsch. u. Statist., 6, 1975, 901-912.

[14] F. Tyndall: A duality theorem for a class of continuous linear programming problems, Journal of SIA M, 13, 1965, 644-666.

[15] J. Zowe, S. Kurcyusz: Regularity and stability for the mathematical programming problem in Banach spaces, Applied Mathematics and Optimization, 5, 1979, 49-62. 